\title{
MICROWAVE APPLICATIONS IN FOOD INDUSTRIES
}

\section{K. SOWJANYA ${ }^{1} \&$ A. ASHOK KUMAR ${ }^{2}$}

${ }^{I}$ Department of Food Science and Technology, CFST, Angrau, Hyderabad, India

${ }^{2}$ Department of Food Engineering, CFST, Angrau, Hyderabad, India

\begin{abstract}
Microwave heating is a process of heating technique, where heating causes the electric field to polarize the charges in the material by two mechanisms namely polarization and conduction. Microwave frequencies have very short wave lengths (33.3 cm at $900 \mathrm{MHz}, 12.2 \mathrm{~cm}$ at $2450 \mathrm{MHz}$ ). This process is based on the ability of a particular substance to absorb microwave energy and effectively convert the electromagnetic energy to heat. Due to mechanical oscillation, the frictional forces within the molecules cause heat to be de

veloped due to the motion of dipoles. Microwave heating includes induction, radio frequency, direct resistance or infra-red heating. Microwaves have many advantages in food processing such as drying, tempering, blanching, cooking, pasteurization, sterilization, and baking. When compared with convectional heating methods, microwave heating has considerable advantages, high reliability, especially with regard to energy efficiency, most efficient in drying within the falling rate period, puffing of a material in case of doughnuts by internal pressure. During microwave heating, heat is transferred from the surface of food to the interior by convection and conduction. By using microwave heating processing of chunks of meat, sardines, and skinless frankfurters can be done. Microwave bread pasteurization is one of the processes which enhances the destruction of micro organisms and heat liable spores during cooling, slicing, and packaging operation. The present study involves microwave applications in different food processing operations.
\end{abstract}

KEYWORDS: Microwave, Induction, Resistance, Infra-Red, Electromagnetic Spectrum \& Etc

Received: Jun 08, 2017; Accepted: Jun 28, 2017; Published: Jul 12, 2017; Paper Id: IJIETAUG20171

\section{INTRODUCTION}

Nowadays, the foods available in the market are produced using conventional thermal processing. The microwave processed foods are shelf stable and have good qualitative traits than foods processed using other conventional thermal processing. The microwave energy has been in industrial processing for many years. This technique was originally conceived about forty years ago. Its adoption against conventional heating methods has been special advantage such as faster throughputs energy savings and quality improvement. The microwave system is composed of magnetron, set- up transformer, oven cavity, wave guide, applicator and a sample to be processed. Microwave frequencies above $500 \mathrm{MHz}$ wired circuits cannot be used where we can observe in the case of radio frequency heating, and the power is transferred to applicator containing the material to be processed in waveguides. In modern industrial microwave heating, systems are used for a diversity of processes in the food industry, tempering, thawing, continuous baking, vacuum drying, pasteurization and sterilization. The overall efficiency of microwave heating systems is usually very high, because of high power magnetrons at the rate of $85 \%$ at $900 \mathrm{MHz}$, $80 \%$ at $2450 \mathrm{MHz}$. 


\section{MICROWAVE APPLICATIONS FOOD INDUSTRIES}

Industrial applications microwave heating is the most effective treatment operation in the food processing industries. The largest application has been seen in defrosting or thawing frozen foods, such as blocks of meat prior to further processing and pasteurization and sterilization of packaged foods. By microwave heating pasteurization is carried out in the range of $75^{\circ} \mathrm{C}$ to $80^{\circ} \mathrm{C}$ to prolong the shelf life approximately four weeks. In addition microwave heating leads to destruction of pathogens or inactivation of enzymes and largely influence on enhancement of thermal effects.

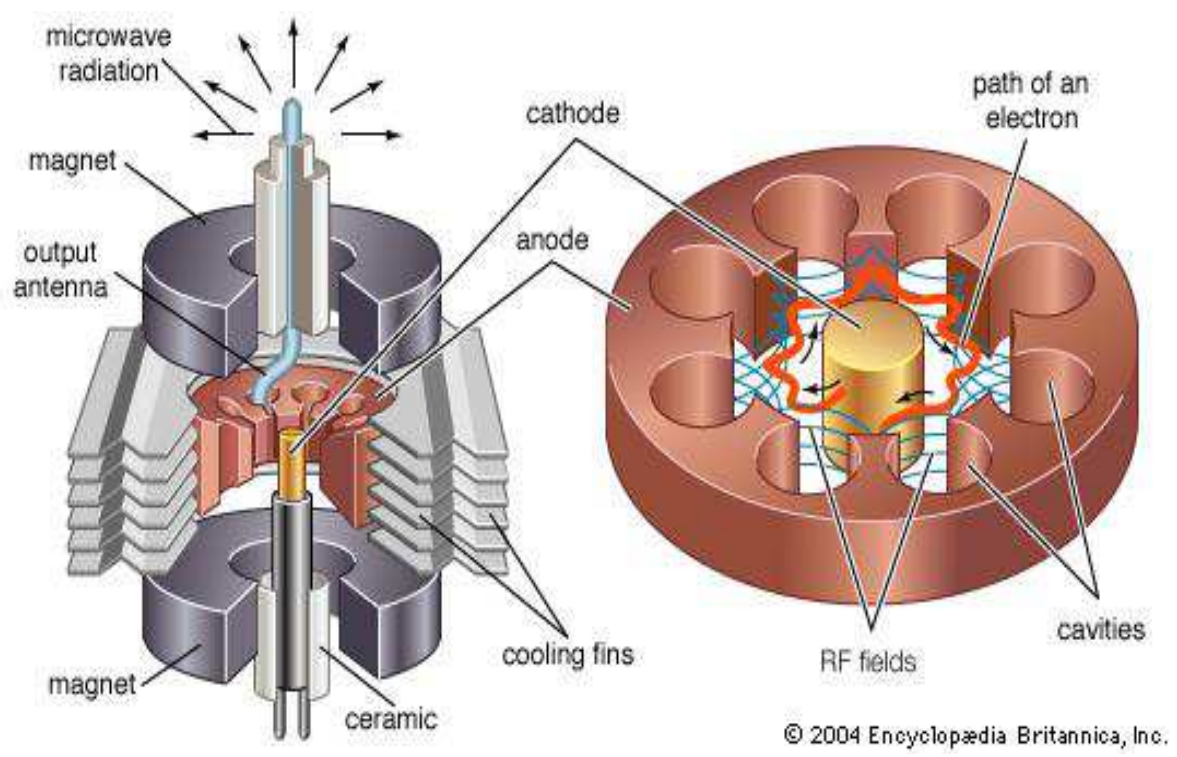

Figure 1: Microwave Heating Equipment

\section{Thawing and Tempering}

During conventional thawing of frozen foods, the lower thermal conductivity of water, compared with ice, reduces the rate of heat transfer and thawing slows as the outer layer of water increases in thickness. Microwaves and radio frequency energy are used to rapidly thaw small portions of food and for melting fats (for example butter, chocolate and fondant cream). However, difficulties arise with larger (e.g. $25 \mathrm{~kg}$ ) frozen blocks (for example egg, meat, and fish and fruit juice) used in industrial processes. Water has a higher loss factor than ice and, as a result, heats rapidly once the ice melts. In the large blocks, thawing does not take place uniformly, and some portions of the food may cook while others remain frozen. This is overcome to some extent by reducing the power and extending the thawing period or by using pulsed microwaves to allow time for temperature equilibration

\section{Baking and Cooking}

By using microwave heating time, energy, space, nutrients are saved during baking. Baking is a complex process where both heat and mass transfer takes simultaneously and causes physical and chemical and biochemical changes like starch gelatinization, liberation of carbon dioxide from leavening agents, volume expansion, evaporation of water, crust formation and browning reactions. Microwave cooking of unfrozen foods can reduce the cooking time and achieve a uniform temperature profile inside foods. The surface temperature of the foods during microwave cooking may rise to $100^{\circ} \mathrm{C}$. Halogen lamp microwave combination baking is new technology in the bakery industry. This halogen lamp heating provides near infrared radiation light with high frequency and low penetration depth. When compared with conventional baking microwave baked products are having the similar specific volume and crust color but firmness of bread are higher. 
Cakes, breads, doughnuts, muffins, biscuits are the examples for edible foams. By using microwave heating in baking heat transfer related problems are overcome. The results like microwave bread baking at $896 \mathrm{MHz}$ using low protein flour improves the gas holding capacity and development of microwave doughnut proofer showed the result of successful usage of microwave heating in baking. By utilizing the microwave technology, doughnuts volumes are increased. The fat absorption can be reduced to $25 \%$ by using the microwave heating; hence, these doughnuts are having longer shelf life better sugar stability, and excellent eating quality. The microwave baking process increases the quality of baked products such as volume, texture, color, porosity, and moisture content. The biscuit which are baked by using microwave heating shows the strong structure and less susceptibility to humid environments.

\section{Dehydration}

Microwaves and radio frequency energy overcome the barrier to heat transfer caused by the low thermal conductivity. This prevents damage to the surface, improves moisture transfer during the later stages of drying and eliminates case hardening. The radiation selectively heats moist areas while leaving dry areas unaffected. It is not necessary to heat large volumes of air, and oxidation by atmospheric oxygen is minimized. However, the higher cost of microwaves and radio frequency units, together with the smaller scale of operation, compared with traditional methods of dehydration, restrict microwave drying to 'finishing' (removing the final moisture) of partly dried or low-moisture foods.

During the drying process control of electromagnetic wave, direction plays an important role. During drying process, those microwaves produce the electromagnetic field internally in the substance and internal pressure gradient pumps the water to surface. Generally done cases of chips and cookies during finish drying. During microwave drying, pressure builds up and evaporation in the interior of the food case increases. This process is used to dry pasta. By using microwaves by various systems, different products are prepared. Pasta, egg yolk powder, tomato paste, chocolate powder and snack food can be prepared by microwave-hot air drying. Fruit juice powder can be prepared by microwave vacuum drying at the range of $2450 \mathrm{MHz}$. Coffee, vegetables, mushrooms are prepared by microwave freeze drying. Potato chip drying and snack food drying are utilizing the microwave drying process. By using microwave treatment at different power levels germination of what grains can be controlled. Microwaves are increasing the performance of heating by developing more heat. Hence microwave drying is a rapid drying technology and most effective in the fields where rapid heating of foods required. In parsley, for example, most essential oils are present as a separate phase with high boiling temperature. For fast drying conditions (high microwave energy input) only the small amount of volatile essential oils that is dissolved is lost, whereas there is not enough time to resolve the remaining oil in the separated phase. In contrast, the retention of water soluble aromas, as in apples, is not as advantageous, since the microwave energy generates many vapor bubbles, so that the volatile aromas have a large surface to evaporate. Nevertheless, the low pressures limit the product temperatures to lower values, as long as a certain amount of free water is present and this helps to retain temperature sensitive substances like vitamins, colors etc. So, in some cases the high quality of the products could make also this relative expensive process economical. Microwave vacuum dehydration is used for the concentration or even powder production of fruit juices and drying of grains in short times without germination. Newly and successfully applied is the combination of pre-air-drying, intermittent microwave vacuum drying (called puffing) and post-air-drying. It is predominantly used to produce dried fruits and vegetables, with improved rehydration properties after the form is stabilized by case hardening due to conventional airdrying, the microwave vacuum process opens the cell structures (puffing) due to the fast vaporization of water and an open pore structure is generated. The subsequent post-drying reduces the water content to the required value. 


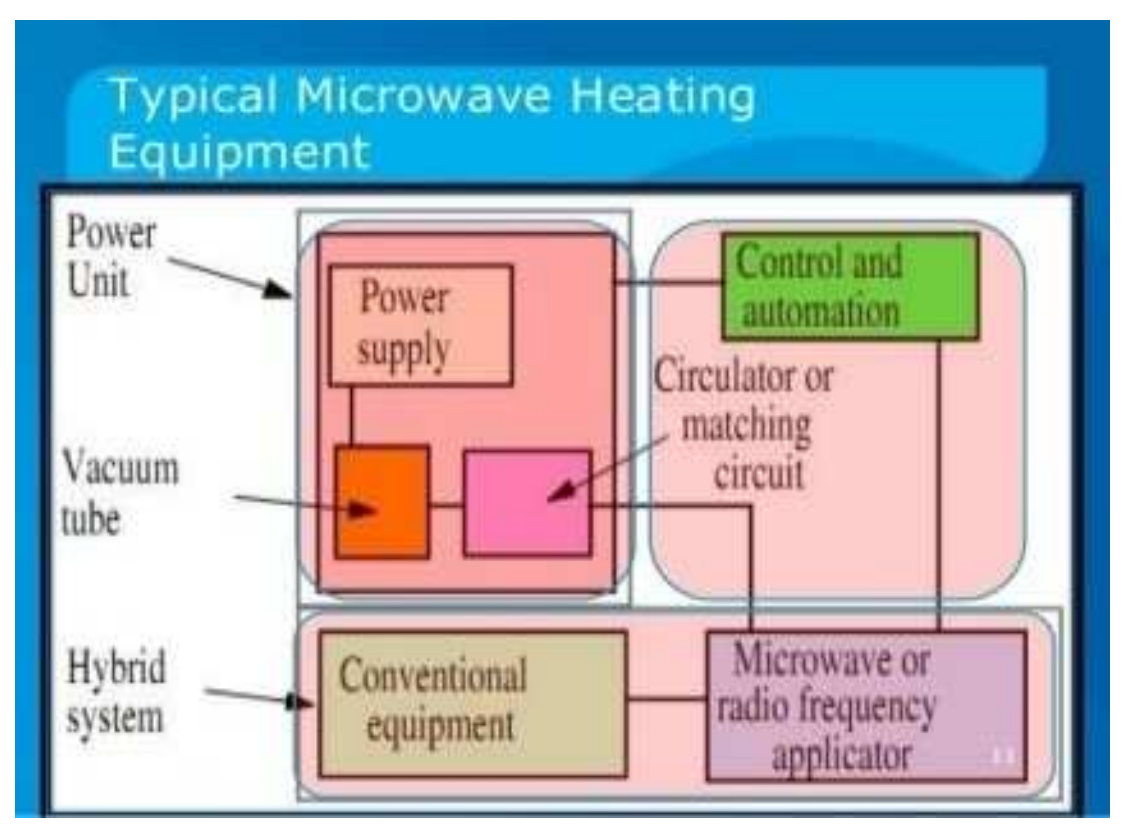

Figure 2

\section{Pasteurization}

Pasteurization of packed, complete pasta meals, soft bakery goods and peeled potatoes by microwaves is reported by Brody (2004). Most systems developed so far involve packaging the products in flat packages using thermoform/vacuum/gas flush seal equipment. Packages are heated in tunnel conveyors, up to $25 \mathrm{~m} \mathrm{long}$, using a combination of microwaves and hot air at $70-90^{\circ} \mathrm{C}$, followed by an equilibration stage where the slowest heating parts of the packs reach $80-85^{\circ} \mathrm{C}$ within $10 \mathrm{~min}$. The packs are then cooled to $1-2^{\circ} \mathrm{C}$ and have a shelf life of approximately 40 days at $8^{\circ} \mathrm{C}$. (T Ohlsson, 2000)

\section{Sterilization}

Sterilization by microwaves is achieved in laminated pouches made from polypropylene/EVOH or PVDC/polypropylene in the Multitherm process. The pouches, which are transparent to microwaves, are formed and filled from a continuous reel of film, but are not separated. This produces a chain of pouches that passes through a continuous hydrostat system, similar to a small hydrostatic steam sterilizer. In this case, the pouches are submerged in a medium that has a higher dielectric constant than the product and heating is by microwaves instead of steam. In a system described by Stenstrom (2003), the product passes through seven liquid baths, heated at up to $90^{\circ} \mathrm{C}$, and the final sterilizing temperature reaches more than $130^{\circ} \mathrm{C}$, before cooling. Both sterilization and pasteurization using microwaves are yet to be widely used in the food industry, but they have the potential to become increasingly important.

\section{QUALITY OF MICROWAVE-DRIED FOOD PRODUCTS}

Effects of microwave on food and nutrients According to Anahad O'Connor (2007) will destroy some nutrients in food, but the key variables are water, time to cook and range of temperature. Some times, over heating the foods cause the unacceptability to consumer which was resulted in non uniform energy absorption and leads to uneven temperature distribution. This temperature distribution affects the sensory attributes, hence the control of heating uniformity is essential step in microwave heating process. The prolonged exposure to high temperature results loss of flavor, color, texture, and overall quality. 


\section{CONCLUSIONS}

The successful applications of microwave heating technology for processing of various foods have been discussed in the present review. (K. M Knutson, 2001). The microwave heating technology for pasteurization and sterilization contributed to destroy pathogenic microorganisms effectively and significantly reduce processing time without serious damage in overall quality of liquid food as compared to traditional methods. The use of microwave heating for food processing applications such as blanching, cooking, and baking has a great effect on the preservation of nutritional quality of food. Furthermore, microwave heating could significantly require less energy consumption for dehydrating food than conventional method. In these days, the potential of continuous flow microwave heating at commercial scale and the combination of heating methods supplemented with conventional thermal treatment for uniform heating of particulate foods has been widely investigated due to inherent advantages of microwave heating. (S. V Lishchuk) Although microwave heating technology for a variety of food processing applications provide significant advantages with respect to lethal effect on pathogens, processing time, and energy consumption, several other quality aspects of food products processed using conventional methods are still better than microwave in terms of color, texture, and other organoleptic properties of food products(J James) Therefore, the investigation of parameters which can influence the workability of microwave heating such as dielectric, physical, and chemical properties of food products should be carried out.

\section{REFERENCES}

1. K. M Knutson, E. H Marth, and M. K Wagner, 1987Microwave Heating of Food', Lebensmittel-Wissenschaft undTechnologie, 20, 101-110,

2. S. V Lishchuk, and J Fischer, 2001Velocity Distribution of Water Molecules in Pores under Microwave Electric Field, International Journal of Thermal Sciences, 40

3. Anahad O'Connor, and R Marc, 2007The microwave processing of foods, Woodhead Publishing Limited and CRC Press, 20312

4. T Ohlsson, 2000Minimal processing of foods with thermal methods', in Barbosa-CaÂnovas $G$ and Gould G, Innovations of Food Processing, Technomic Publishing, Lancaster, PA.

5. J. J Rodriaguez, Barbosa-Caa novas, G. V., Gutiearrez-Loa Pez. G. F., Dorantes-Alvarez, L., Won-Yeom, H., and Zhang, H. Q. (2003An update on some key alternative food processing technologies: microwave, pulsed electric field, high hydrostatic pressure, irradiation and ultrasound', in GutieÂrrez-LoÂpez G F and Barbosa-CaÂnovas G V, Food Science and Food Biotechnology, CRC Press, Washington D.C., 279304

6. Occupational Safety \& Health Administration (OSHA) United States Department of Labor. Copyright $\odot 2012$

7. J James, and C James, 2002Thawing and tempering. In Meat Refrigeration, Woodhead Publishing, Cambridge, 159190 
